\title{
Consultores ad hoc da Rodriguésia em 2005
}

\author{
Agnes Elisete Luchi \\ Alessandro Rapini \\ Alexandre Adalardo de 0 liveira \\ André Luís Laforga Vanzela \\ André Mantovani \\ Andrea Ferreira da Costa \\ Andrea Pereira Luizi Ponzo \\ Aparecida Donisete Faria \\ Bernardo Antonio Perez da G ama \\ Cassia Mônica Sakuragui \\ Catarina Carvalho Nievola \\ Cíntia Kameyama \\ Claudia Petean Bove \\ Claudine Massi Mynssen \\ Dan Nicolson \\ Daniela Zappi \\ Daniela G uimarães Simão \\ Dória Maria Saiter Gomes \\ Dorothy Sue Dunn de Araújo \\ Douglas Antônio de Carvalho \\ Eduardo $\mathrm{G}$ omes $\mathrm{G}$ onçalves \\ Eliana Regina Forni Martins \\ Elisabeth Atalla Mansur de 0 liveira \\ Elsie Franklin G uimarães \\ Fernanda Reinert Thomé Macrae \\ Fernando Pedroni \\ Flávio Coelho Edler \\ G erlene Lopes Esteves \\ J efferson Prado \\ João Renato Stehmann \\ John Du Vall Hay \\ José Aldo Alves Pereira \\ Júlio Antônio Lombardi
}

\author{
Katia Cavalcanti Pôrto \\ Leandro Freitas \\ Lidyanne Yuriko Saleme Aona \\ Lucia G arcez Lohmann \\ Marco Aurélio Leite Fontes \\ Marcus Alberto Nadruz Coelho \\ Maria Cândida Henrique Mamede \\ Maria das G raças Sajo \\ Maria do Carmo Estanislau do Amaral \\ Marli Pires Morim \\ Mauro G aletti Rodrigues \\ Mercedes Maria da Cunha Bustamante \\ Milton G roppo Júnior \\ Nair Sumie Yokoya \\ Nidia Majerowicz \\ 0 berdan J o sé Pereira \\ Pablo J osé Francisco Pena \\ Patrícia Borges Pita \\ Paulo Takeo Sano \\ Paul J. M. Maas \\ Regina Helena Potsch Andreata \\ Ricardo Pereira Louro \\ Ricardo Tadeu de Faria \\ Roberto Campos Villaça \\ Ronald Bastos Freire \\ Rosana Romero \\ Thomas B. Croat \\ Timothy Molton \\ Vânia Regina Pivello \\ Vera Lúcia Scatena \\ Waldir Mantovani \\ Yule Ro berta Ferreira N unes
}


